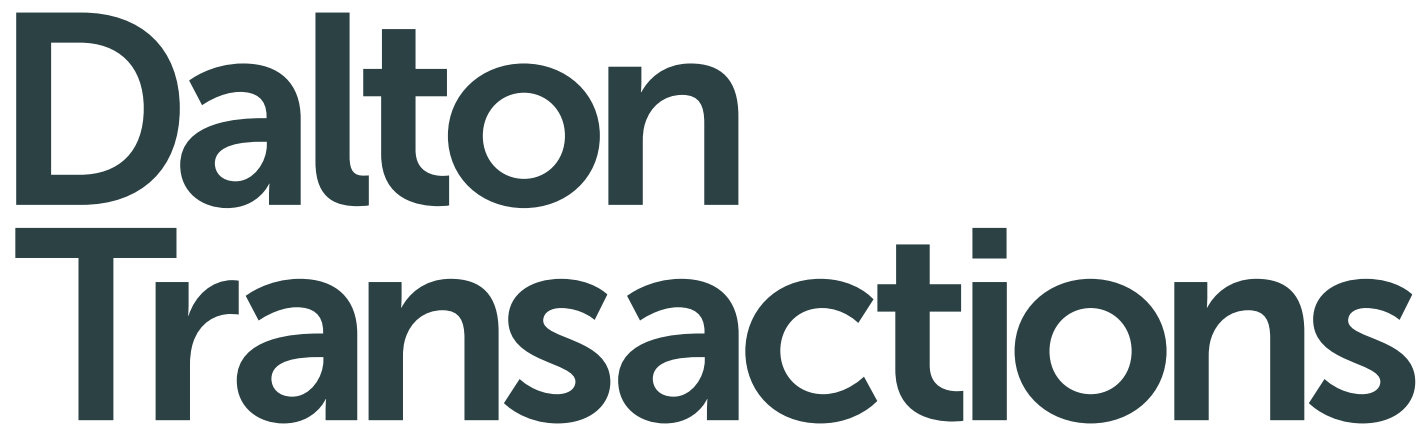

An international journal of inorganic chemistry www.rsc.org/dalton

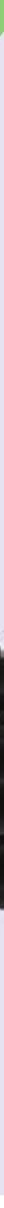

ISSN 1477-9226

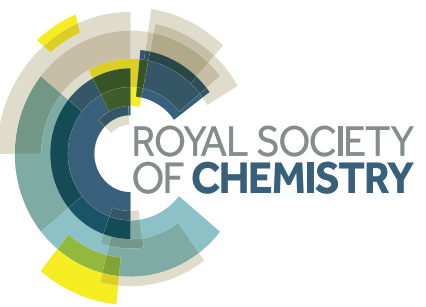

\title{
COMMUNICATION
}

David Klar et al.

Hysteretic behaviour in a vacuum deposited submonolayer of single ion magnets 


\section{Hysteretic behaviour in a vacuum deposited submonolayer of single ion magnets $\uparrow$}

10686

Received 4th April 2014,

Accepted 12th May 2014

DOI: $10.1039 / c 4 d t 01005 a$

www.rsc.org/dalton

With element-specific X-ray absorption spectroscopy and X-ray magnetic circular dichroism we have investigated submonolayer coverages of $\mathrm{TbPc}_{2}$ and $\mathrm{DyPc}_{2}$ molecules sublimated on highly ordered pyrolytic graphite. We have studied the field dependence of the magnetization of the central lanthanide ion at very low temperatures. Even in zero applied magnetic field we still observe a remanence in the magnetization. Since there are neither intermolecular coupling nor magnetic interactions with the substrate, this remanent behaviour results just from single-ion anisotropy. On the very inert surface of graphite at temperatures between $0.5 \mathrm{~K}$ and $2 \mathrm{~K}$ the spin relaxation is slow enough to observe a memory effect in the timescale of the experimental measurements.

Integrating organic materials into spintronic devices has become an important part of research over the last few years. ${ }^{1-6}$ Organic molecules offer new technological possibilities concerning small and effective data storage devices. Single molecule magnets (SMMs) are predestined for such very small devices because of their magnetic hysteresis without the need for long range ordering and have been the subject of intense studies over the last two decades. Most of the SMMs consist of several magnetic ions, e.g. $\mathrm{Mn}_{12}$ complexes, ${ }^{7-9}$ while in bis(phthalocyaninato) lanthanide complexes $\left(\mathrm{LnPc}_{2}\right)$ there is only a single $\mathrm{Ln}$ ion $\left(\mathrm{Dy}^{3+}, \mathrm{Tb}^{3+}\right)$ centered at the molecule between two phthalocyanine (Pc) rings (Fig. 1c). The remanent magnetization arises from the single ion anisotropy. ${ }^{10-12}$ Both molecules show uniaxial anisotropy, with the energy barriers of

\footnotetext{
${ }^{a}$ Faculty of Physics and Center for Nanointegration Duisburg-Essen (CENIDE), University of Duisburg-Essen, Lotharstraße 1, D-47048 Duisburg, Germany. E-mail: david.klar@uni-due.de; Fax: +49 (o)203 379 3601; Tel: +49 (0)203 3791662 ${ }^{b}$ Centro S3, Istituto Nanoscienze - CNR, via Campi 213/a, 41125 Modena, Italy ${ }^{c}$ Institut de Physique et Chimie des Matériaux de Strasbourg, UMR 7504 UdS-CNRS, 67034 Strasbourg Cedex 2, France

${ }^{d}$ Institute of Nanotechnology, Karlsruhe Institute of Technology (KIT), D-76344 Eggenstein-Leopoldshafen, Germany

${ }^{e}$ Synchrotron SOLEIL, L'Orme des Merisiers, Saint-Aubin - BP 48, 91192 Gif-surYvette, France

$\dagger$ Electronic supplementary information (ESI) available: Bulk reference measurements and characterization of the molecules and the submonolayer. See DOI: 10.1039/c4dt01005a
}

$\mathrm{TbPc}_{2}$ being much higher than that of the DyPc $\mathrm{P}_{2}$ molecule. Spin and charge dynamics of powder samples of these two molecules were studied by Branzoli et al. ${ }^{13}$ The general problem with regard to applications in devices is the conservation of their SMM behaviour at high enough temperatures. The spin relaxation of $\mathrm{TbPc}_{2}$ molecules above $4 \mathrm{~K}$ is already too fast to detect a remanent magnetization by X-ray absorption spectroscopy (XAS), a rather slow experimental method. ${ }^{14}$ Magnetization curves of $\mathrm{TbPc}_{2}$ molecules show typically butterfly shaped hysteresis even at very low temperatures, originating from the spin quantum tunneling at zero magnetic fields. ${ }^{15-17}$ At temperatures below $4 \mathrm{~K}$ the relaxation is slow enough to identify these quantum tunneling effects with ac susceptibility measurements. ${ }^{18}$ One possibility to block relaxation at higher temperatures is the coupling on a ferromagnetic substrate. ${ }^{19,20}$ The magnetic anisotropy and field dependence of $\mathrm{TbPc}_{2}$ molecules have been investigated by XAS on non-magnetic metallic surfaces, ${ }^{14,17}$ on antiferromagnetic surfaces ${ }^{21}$ and ferromagnetic surfaces. ${ }^{22}$ Here we investigate submonolayer coverages of $\mathrm{TbPc}_{2}$ and $\mathrm{DyPc}_{2}$ molecules on highly ordered pyrolytic graphite (HOPG) with XAS, and in particular with X-ray magnetic circular dichroism (XMCD). Over the last decade, XAS and XMCD have been successfully used as powerful methods to determine the electronic and magnetic properties of submonolayers of different magnetic molecules interacting with surfaces. ${ }^{23-27}$ The deposition of less than a monolayer leads to well separated and oriented molecules on the graphite surface. In contrast to a metallic surface the hybridization between the molecules and the graphite is very weak and this enables the observation of the undisturbed molecules' properties. Recently it has been demonstrated that the $\mathrm{TbPc}_{2}$ molecules are strongly affected by their environment ${ }^{28}$ : the opening of the magnetization loop disappears on a conducting substrate, whereas remanence appears in bulk systems at $T=2 \mathrm{~K}$ (see also ESI $\dagger$ ). We show here that at temperatures between $0.5 \mathrm{~K}$ and $2 \mathrm{~K}$ the spin relaxation is slow enough to observe a completely open magnetization curve with only slight butterfly shape. At $0.5 \mathrm{~K}$ we are able to observe remanence even for $\mathrm{DyPc}_{2}$, where the blocking temperature is 

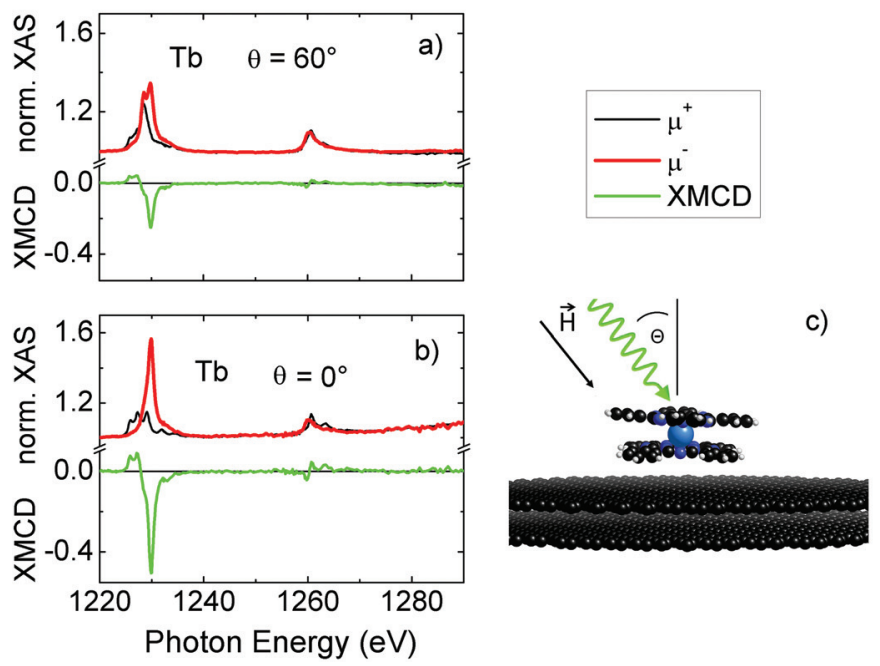

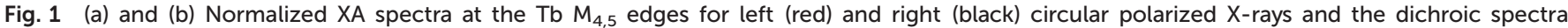

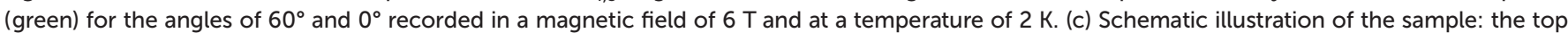

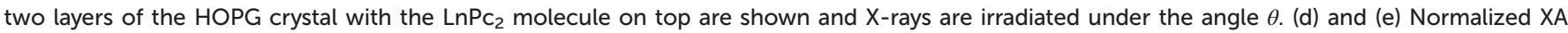

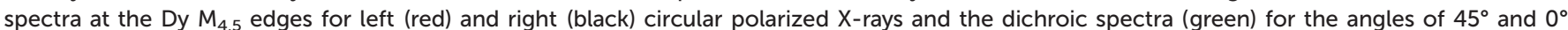
recorded in a magnetic field of $1 \mathrm{~T}$ and at a temperature of $0.5 \mathrm{~K}$.

reported to be considerably lower than for $\mathrm{TbPc}_{2} \cdot{ }^{18}$ By timedependent measurements of the magnetization we could determine that the relaxation time at this temperature is of the order of one hour and therefore detectable by our experimental technique.

DyPc $c_{2}$ and $\mathrm{TbPc}_{2}$ were synthesized according to an earlier established procedure ${ }^{13}$ and analytical data confirm their intact structure (see $\mathrm{ESI}^{\dagger}$ ). A submonolayer $(0.5 \pm 0.2 \mathrm{ML})$ coverage of $\mathrm{LnPc}_{2}$ was prepared in situ by thermal evaporation of the molecules onto the freshly cleaved HOPG crystal held at room temperature (for characterization details, see ESI $\dagger$ ). The measurements relative to the $\mathrm{TbPc}_{2}$ molecule were performed at the DEIMOS ${ }^{29}$ beamline at the synchrotron SOLEIL, while $\mathrm{DyPc}_{2}$ molecules were studied at the SIM beamline at the SLS synchrotron, Paul Scherrer Institute, Villigen, Switzerland with the unique possibility to perform XMCD experiments at subKelvin temperatures. ${ }^{30}$ The temperature of $0.5 \mathrm{~K}$ is crucial for the detection of the open hysteresis curve of $\mathrm{DyPc}_{2}$ because of its very low blocking temperature. Field-dependent measurements were performed to determine the SMM properties of the molecules. To study the spin relaxation after turning off the magnetic field, we carried out time-dependent XMCD measurements. With angle-dependent XMCD we studied the molecule orientation and the magnetic anisotropy.

Fig. 1 shows the angle-dependent spectra at the $\mathrm{Tb}$ and the Dy $\mathbf{M}_{4,5}$ edges for different X-ray incidence angles, normal $\left(0^{\circ}\right)$ and grazing $\left(60^{\circ}\right.$ or $\left.45^{\circ}\right)$ at $T=2 \mathrm{~K}$ or $0.5 \mathrm{~K}$ and external magnetic field $B=6 \mathrm{~T}$ or $1 \mathrm{~T}$. The black line represents the circular right polarization $\mu^{+}$, the red line the circular left polarization $\mu^{-}$and the green line the difference of the two spectra, the XMCD signal. The integrated white line $\left(\mu^{+}+\mu^{-}\right) / 2$ intensity at the $\mathrm{M}_{5}$ edge is normalized to 1 . This normalization enables a direct comparison of the magnitude of the XMCD signals corresponding to the different angles. In the recorded spectra there are clear structural differences visible, especially at the $\mathrm{M}_{5}$ edge. However, the shape of the XMCD does not change with variation of the angle. Only the magnitude of the XMCD changes exactly with the cosine of the incidence angle. This behaviour of the magnetic anisotropy was observed for the $\mathrm{TbPc}_{2}$ molecules also on other substrates. ${ }^{14}$ The large anisotropy prevents the magnetic moments of $\mathrm{Tb}$ from turning into the plane of the Pc ring. The magnetization of the $\mathrm{LnPc}_{2}$ is still perpendicular, even in an external magnetic field of $6 \mathrm{~T}$ applied in the Pc-plane direction. Since we detect the magnetization just along the incident photons, the spectra only give the projection of the magnetization that is scaled with $\cos (\theta)$.

The field dependence of the $\mathrm{Tb}$ magnetization is given in Fig. 2(a). The curves show the maximum of the $\mathbf{M}_{5}$ edge $(1229.8 \mathrm{eV})$ normalized to the pre-edge $(1220 \mathrm{eV})$. At a temperature of $2 \mathrm{~K}$ the magnetization of the $\mathrm{Tb}$ ion reaches the saturation value at about $1 \mathrm{~T}$. Between the positive and negative saturation the magnetization curve is clearly open. The inset in this figure demonstrates that the curves do not cross at zero field. There is a remanent signal with a small coercive field of about $50 \mathrm{mT}$. The measurement of one complete curve takes more than one hour, but the relaxation at this temperature is slow enough to observe the remanent magnetization. The spin quantum tunneling that causes the typical butterfly shape is strongly reduced, but apparently the hysteresis curve is a bit more narrow at $B=0 \mathrm{~T}$, indicating that spin tunneling is still present. The detailed origin of this behaviour is still unclear and falls beyond the scope of the present work. We note that a similar behaviour has been recently reported by another group for a crystallite sample of the same molecule. ${ }^{15}$ The spin relaxation is very sensitive to the temperature, leading to a vanishing remanence above $4 \mathrm{~K}$. 

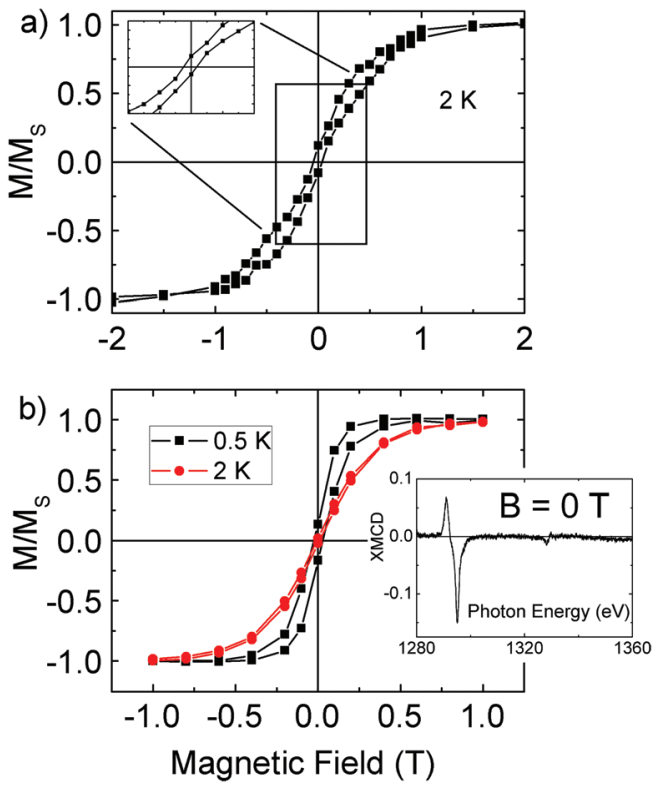

Fig. 2 Field-dependent magnetization measured at the maximum of (a) the $\mathrm{Tb} \mathrm{M}_{5}$ edge XMCD at $T=2 \mathrm{~K}$ and (b) the Dy $M_{5} \mathrm{XMCD}$ edge at $T=$ $0.5 \mathrm{~K}$ (black) and $T=2 \mathrm{~K}$ (red), normalized to the saturation magnetization.

Each curve in Fig. 2(b), showing the field dependence of the Dy magnetization, consists of only 26 data points. Each point belongs to an XMCD maximum at $1295.1 \mathrm{eV}$, taken from completely recorded $\mu^{+}$and $\mu^{-}$spectra. In this way, each data point took approximately 30 minutes. Nevertheless, the opening of the magnetization curve is clearly visible for the black magnetization curve, demonstrating the long relaxation times at this low temperature. The remanent magnetization is also verified by the XMCD signal (Fig. 2b, inset) at $B=0 \mathrm{~T}$. However, at $T=$ $2 \mathrm{~K}$ (red line), no remanent magnetization is visible, confirming the lower blocking temperature of $\mathrm{DyPc}_{2}$ compared to $\mathrm{TbPc}_{2}$.

To make a quantitative statement about the relaxation time of the $\mathrm{DyPc}_{2}$ magnetization, we measured the time-dependent magnetization without an applied magnetic field (Fig. 3). Each triangle in this figure represents the normalized difference

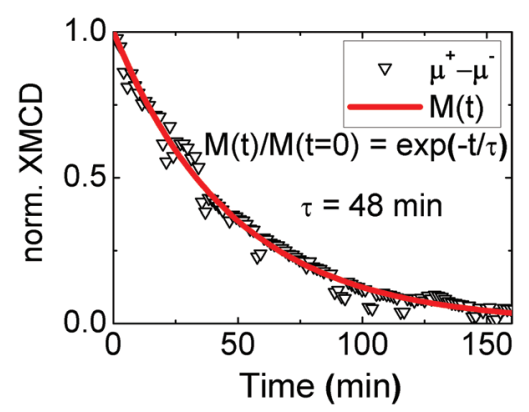

Fig. 3 Time dependence of the normalized XMCD intensity at the maximum of the Dy $M_{5}$ edge without external magnetic field at a temperature of $0.5 \mathrm{~K}$. The red curve is an exponential function with a half life of 48 minutes. $\mu^{+}-\mu^{-}$at the maximum XMCD intensity of $1295.1 \mathrm{eV}$. Approximately one data point was recorded every 90 seconds. The functional correlation between the XMCD intensity and time was fitted with a simple exponential function, while the maximum at the beginning was normalized to 1 . The red curve fits the data points very well, demonstrating that the relaxation follows an exponential behaviour with a half-life of $\tau=$ 48 minutes. This time is in the range of typical XMCD measurements, giving us the opportunity to observe the magnetic remanence.

In conclusion, we have observed the remanent magnetization of both $\mathrm{TbPc}_{2}$ and $\mathrm{DyPc}_{2}$ molecules. Submonolayers of these molecules on the very inert HOPG substrate were deposited under UHV conditions to ensure that the interaction with the molecules' environment is kept as low as possible. For the first time we could show the open hysteresis of vacuum deposited and well oriented $\mathrm{LnPc}_{2}$ molecules in an X-ray absorption study due to the very low temperature at which the measurements have been performed. This is a fundamental step for the possibility to use molecular memories in future devices. Because of the strongly suppressed spin relaxation at this temperature we were also able to determine a half life of the magnetization of 48 minutes.

Experiments were performed on the DEIMOS beamline at SOLEIL Synchrotron, France (proposal number 20110125) and on the SIM BEAMLINE (proposal number 20110200) at the Swiss Light Source, Paul Scherrer Institute, Villigen, Switzerland. We are grateful to the SOLEIL staff for smoothly running the facility. We thank Armin Kleibert at Swiss Light Source whose outstanding efforts have made these experiments possible.

\section{References}

1 A. R. Rocha, V. M. Garcia-Suarez, S. W. Bailey, C. J. Lambert, J. Ferrer and S. Sanvito, Nat. Mater., 2005, 4, 335-339.

2 L. Bogani and W. Wernsdorfer, Nat. Mater., 2008, 7, 179.

3 M. Mannini, F. Pineider, P. Sainctavit, C. Danieli, E. Otero, C. Sciancalepore, A. M. Talarico, M.-A. Arrio, A. Cornia, D. Gatteschi, et al., Nat. Mater., 2009, 8, 194-197.

4 A. Candini, S. Klyatskaya, M. Ruben, W. Wernsdorfer and M. Affronte, Nano Lett., 2011, 11, 2634-2639.

5 M. Urdampilleta, S. Klyatskaya, J. Cleuziou, M. Ruben and W. Wernsdorfer, Nat. Mater., 2011, 10, 502-506.

6 R. Vincent, S. Klyatskaya, M. Ruben, W. Wernsdorfer and F. Balestro, Nature, 2012, 488, 357-360.

7 R. Sessoli, D. Gatteschi, A. Caneschi and M. Novak, Nature, 1993, 365, 141-143.

8 G. Christou, D. Gatteschi, D. N. Hendrickson and R. Sessoli, MRS Bull., 2000, 67.

9 D. Gatteschi and R. Sessoli, Angew. Chem., Int. Ed., 2003, 42, 268-297.

10 J. D. Rinehart and J. R. Long, Chem. Sci., 2011, 2, 20782085. 
11 N. Ishikawa, M. Sugita, T. Okubo, N. Tanaka, T. Iino and Y. Kaizu, Inorg. Chem., 2003, 42, 2440-2446.

12 F. Branzoli, P. Carretta, M. Filibian, G. Zoppellaro, M. J. Graf, J. R. Galan-Mascaros, O. Fuhr, S. Brink and M. Ruben, J. Am. Chem. Soc., 2009, 131, 4387-4396.

13 F. Branzoli, P. Carretta, M. Filibian, M. J. Graf, S. Klyatskaya, M. Ruben, F. Coneri and P. Dhakal, Phys. Rev. B: Condens. Matter, 2010, 82, 134401.

14 S. Stepanow, J. Honolka, P. Gambardella, L. Vitali, N. Abdurakhmanova, T.-C. Tseng, S. Rauschenbach, S. L. Tait, V. Sessi, S. Klyatskaya, et al., J. Am. Chem. Soc., 2010, 132, 11900-11901.

15 M. Gonidec, E. S. Davies, J. McMaster, D. B. Amabilino and J. Veciana, J. Am. Chem. Soc., 2010, 132, 1756-1757.

16 M. Gonidec, R. Biagi, V. Corradini, F. Moro, V. De Renzi, U. del Pennino, D. Summa, L. Muccioli, C. Zannoni, D. B. Amabilino, et al., J. Am. Chem. Soc., 2011, 133, 66036612.

17 L. Margheriti, D. Chiappe, M. Mannini, P. Car, P. Sainctavit, M. A. Arrio, F. B. de Mongeot, J. C. Cezar, F. M. Piras, A. Magnani, E. Otero, A. Caneschi and R. Sessoli, Adv. Mater., 2010, 22, 5488.

18 N. Ishikawa, M. Sugita and W. Wernsdorfer, Angew. Chem., Int. Ed., 2005, 44, 2931-2935.

19 A. Lodi Rizzini, C. Krull, T. Balashov, J. Kavich, A. Mugarza, P. Miedema, P. Thakur, V. Sessi, S. Klyatskaya, M. Ruben, S. Stepanow and P. Gambardella, Phys. Rev. Lett., 2011, 107, 177205.

20 D. Klar, S. Klyatskaya, A. Candini, B. Krumme, K. Kummer, P. Ohresser, V. Corradini, V. de Renzi, R. Biagi, L. Joly, J.-P. Kappler, U. del Pennino, M. Affronte, H. Wende and M. Ruben, Beilstein J. Nanotechnol., 2013, 4, 320-324.
21 A. Lodi Rizzini, C. Krull, T. Balashov, A. Mugarza, C. Nistor, F. Yakhou, V. Sessi, S. Klyatskaya, M. Ruben, S. Stepanow, et al., Nano Lett., 2012, 12, 5703-5707.

22 L. Malavolti, L. Poggini, L. Margheriti, D. Chiappe, P. Graziosi, B. Cortigiani, V. Lanzilotto, F. B. de Mongeot, P. Ohresser, E. Otero, et al., Chem. Commun., 2013, 49, 11506-11508.

23 A. Scheybal, T. Ramsvik, R. Bertschinger, M. Putero, F. Nolting and T. A. Jung, Chem. Phys. Lett., 2005, 411, 214.

24 H. Wende, M. Bernien, J. Luo, C. Sorg, N. Ponpandian, J. Kurde, J. Miguel, M. Piantek, X. Xu, P. Eckhold, W. Kuch, K. Baberschke, P. M. Panchmatia, B. Sanyal, P. M. Oppeneer and O. Eriksson, Nat. Mater., 2007, 6, 516.

25 M. Bernien, J. Miguel, C. Weis, M. E. Ali, J. Kurde, B. Krumme, P. M. Panchmatia, B. Sanyal, M. Piantek, P. Srivastava, K. Baberschke, P. M. Oppeneer, O. Eriksson, W. Kuch and H. Wende, Phys. Rev. Lett., 2009, 102, 047202.

26 M. Bernien, D. Wiedemann, C. F. Hermanns, A. Krüger, D. Rolf, W. Kroener, P. Müller, A. Grohmann and W. Kuch, J. Phys. Chem. Lett., 2012, 3, 3431-3434.

27 D. Klar, B. Brena, H. Herper, S. Bhandary, C. Weis, B. Krumme, C. Schmitz-Antoniak, B. Sanyal, O. Eriksson and H. Wende, Phys. Rev. B: Condens. Matter, 2013, 88, 224424.

28 L. Malavolti, M. Mannini, P.-E. Car, G. Campo, F. Pineider and R. Sessoli, J. Mater. Chem. C, 2013, 1, 2935-2942.

29 P. Ohresser, E. Otero, F. Choueikani, K. Chen, S. Stanescu, F. Deschamps, T. Moreno, F. Polack, B. Lagarde, J.-P. Daguerre, et al., Rev. Sci. Instrum., 2014, 85, 013106.

30 I. Letard, P. Sainctavit, J.-P. Kappler, P. Ghigna, D. Gatteschi, B. Doddi, et al., J. Appl. Phys., 2007, 101, 113920-113920. 